\title{
Papel del estrés oxidativo en la infección por SAR-coV-2, y uso de antioxidantes como mecanismo de prevención: una revisión narrativa
}

\author{
Role of oxidative stress in SAR-coV-2 infection, and use of antioxidants as a \\ prevention mechanism: a narrative review
}

\author{
Max Roger Bautista-Leon ${ }^{a}$,Ernesto Alanís-García ${ }^{b}$,Nelly del Socorro Cruz-Cansino ${ }^{c}$, Luis \\ Delgado-Olivares ${ }^{d}$
}

\begin{abstract}
:
The pandemic caused by COVID-19 is seriously affecting the economy, social life, as well as the physical and mental health of the whole world, mainly of people who have a non-communicable disease where oxidative stress is involved. The objective of this paper is to review the literature on the role of oxidative stress in SAR-coV-2 infection and show the effect of antioxidants used in the treatment of COVID-19 and their possible use in the prevention of the infection of this new virus. Because it is a disease of recent appearance, new information appears every day. An analysis of the bibliographic evidence available on the internet was carried out by consulting PubMed and Google Scholar. The analysis of the information obtained revealed that oxidative stress is involved in the infection processes through an epigenetic mechanism that promotes the expression of the ACE2 protein. This protein is the key for the virus to infect the cell, causing an increase in the cellular oxidative state, damaging the structures and functions of the cell, which results in apoptosis and irreversible damage to the tissues. Within the above framework, the use of antioxidants for the treatment of viral infection has been proposed. We conclude that a balanced diet rich in fruits and vegetables can strengthen the immune system and provide the necessary antioxidants to prevent COVID-19.
\end{abstract}

Keywords:

Antioxidants, COVID-19, Epigenome, Oxidative stress, ACE2 protein.

\section{Resumen:}

La pandemia provocada por COVID-19, está afectando gravemente la economía, vida social, así como la salud física y mental de todo el mundo, principalmente de personas que presentan alguna enfermedad no transmisible donde el estrés oxidativo ésta involucrado. El objetivo del presente artículo es hacer una revisión en la literatura sobre el papel que tiene el estrés oxidativo, en la infección de SAR-coV-2 y mostrar el efecto de los antioxidantes utilizados en el tratamiento del COVID-19 y su posible uso en la prevención de la las infección de éste nuevo virus. Debido a que es una enfermedad de reciente aparición, cada día aparece nueva información. Se realizó un análisis de la evidencia bibliográfica disponible en internet consultando PubMed y Google Académico. El análisis de la información obtenida reveló que el estrés oxidativo se encuentra involucrado en los procesos de infección, mediante un mecanismo epigenético que promueve la expresión de la proteína ACE2. Esta proteína es la clave para que el virus infecte a la célula, provocando un incremento del estado oxidativo celular, dañando las estructuras y funciones de la misma, lo cual resulta en apoptosis y daño irreversible a los tejidos. Dentro del marco anterior, se ha propuesto el uso de antioxidantes para el tratamiento de la infección viral. Nosotros concluimos que una alimentación balanceada, rica en frutas y verduras puede fortalecer el sistema inmune y dotar de los antioxidantes necesarios para prevenir la COVID-19.

Palabras Clave:

Antioxidantes, COVID-19, Epigenoma, Estrés oxidativo, Proteína ACE2

\footnotetext{
a Universidad Autónoma del Estado de Hidalgo, Email: megacarnivor@gmail.com

b Universidad Autónoma del Estado de Hidalgo, Email: ealanisg70@yahoo.com.mx

Autor de Correspondencia, Universidad Autónoma del Estado de Hidalgo, https://orcid.org/0000-0002-6771-3684, Email: ncruz@uaeh.edu.mx

d Autor de Correspondencia, Universidad Autónoma del Estado de Hidalgo, https://orcid.org/0000-0002-3506-8393, Email: ldelgado@uaeh.edu.mx
} 


\section{Introducción}

A finales del 2019, en la ciudad de Wuhan en China apareció el virus SAR-coV-2 (Severe Acute Respiratory Syndrome Coronavirus 2), causante del síndrome respiratorio agudo severo o también llamado COVID-19 (por sus siglas en inglés, Coronavirus Disease 2019, respectivamente), el cual debido a su rápida propagación y grado de mortalidad marcó el comienzo de una pandemia a nivel mundial. La cual está afectando la vida socioeconómica, así como la salud física y mental de la población a nivel mundial ${ }^{1}$.

Si bien, todas las personas independientemente de su edad o condición social, tienen el riesgo de contraer COVID-19, existen ciertos sectores de la población que son más propensos al contagio y a la muerte por esta enfermedad. De acuerdo con el departamento de salud y servicios humanos de Estados Unidos ${ }^{2}$, las personas que tienen un mayor de riesgo de infección son aquellas que presentan enfermedades no transmisibles (ENT), así como las que se encuentran en cierto estado fisiológico como el envejecimiento o embarazo.

Tanto en las enfermedades como en las condiciones mencionadas, existe un incremento en la producción de especies reactivas de oxígeno (ERO), que conduce a un estado de estrés oxidativo (EOx), alternado la estructura y función de las diferentes biomoléculas, por lo que los procesos celulares no son realizados correctamente ${ }^{3,4}$. Existen diferentes condiciones que producen EOx, como la edad, la nutrición y las mismas ENT ${ }^{5}$.

Existe una relación entre el EOx y la muerte por COVID19, por lo que las personas mayores de 65 años con alguna ENT, al presentar mayor EOx, tienen un mayor riesgo de infección y muerte por COVID-19, que las personas de menor edad con las mismas enfermedades 6.

De acuerdo con la OMS ${ }^{7}$, hasta el 18 de febrero del 2021 se habían reportado $109,594,835$ casos confirmados de COVID-19, con 2,424,060 muertes en todo el mundo. El análisis de los 20 países con más casos acumulados, muestra que Estados Unidos tiene el primer lugar con $27,491,574$ casos confirmados, así como el primer lugar con 484,379 muertes, con una probabilidad del $1.76 \%$ de fallecer por esta enfermedad. Mientras que México, aunque ocupa el treceavo lugar con 1,806,849 de casos y el tercer lugar con 175,986 muertes acumuladas, tiene la tasa más alta $(8.78 \%)$ de muertes por COVID-19 (Tabla 1). Aún si se toman en cuenta todos los países reportados por la OMS, nuestro país se ubica en el segundo lugar con mayor probabilidad de muerte, encontrándose solo por debajo de Yemen que presenta el 29\% de muertes por COVID-19 (dato no mostrado). Lo puede ser debido a que en México existe una alta prevalencia de $\mathrm{ENT}^{8}$, debido principalmente a los malos hábitos alimenticios de la población mexicana, donde existe un alto consumo de dietas ricas en azúcares refinados y grasas, lo que promueve el desarrollo del EOx y de las ENT, lo que la hace más susceptible al desarrollo del COVID-19. Por lo tanto, la dieta puede ser un factor clave en la prevención del COVID-19. Por lo que, el objetivo del presente documento fue hacer una revisión en la literatura sobre el papel del EOx en la infección de
SAR-coV-2 y analizar el uso de los antioxidantes en el tratamiento y prevención de las infecciones de éste nuevo virus. Debido a que es una enfermedad reciente y cada día aparece nueva información, se realizó un análisis de la evidencia bibliográfica disponible en internet consultando PubMed y Google Académico.

\section{Población en riesgo}

Las ENT, también llamadas enfermedades crónicas, son aquellas que no se transmiten de persona a persona y presentan efectos prolongados sobre la salud con una recuperación muy lenta, por lo que son responsables de la muerte de millones de personas en todo el mundo ${ }^{4,9}$. En este grupo de enfermedades se encuentra la diabetes mellitus, obesidad, inmunodepresión, enfermedades cardiacas, cáncer y afecciones pulmonares ${ }^{9}$. Se ha reportado que, debido principalmente a la predisposición genética y hábitos alimenticios, la probabilidad de desarrollar alguna ENT es mayor en la población de color, la latina y otras etnias ${ }^{10,11}$. De acuerdo con la Encuesta nacional de salud y nutrición (ENSANUT) 2018, en México, las ENT con mayor prevalencia en la población adulta son obesidad (36.1\%), hipertensión (18.4\%) y diabetes (10.3\%) ${ }^{12}$.

Durante el desarrollo de las ENT, se induce la activación de citocinas proinflamatorias que perdura durante todo el tiempo que dura la enfermedad, produciendo un estado inflamatorio debajo grado, el cual sufre un aumento conforme la enfermedad se va agravando ${ }^{4}$. Esto produce un incremento en la producción de ERO, provocando un estado de estrés oxidativo crónico, extendiendo el daño a órganos y tejidos ${ }^{13}$.

\section{Estrés oxidativo}

Al incrementarse los niveles celulares de las ERO, se produce un desbalance entre estas moléculas y los antioxidantes dando lugar a un fenómeno conocido como EOx, el cual modifica la estructura de las diferentes biomoléculas. Las ERO actúan oxidando a los aminoácidos, produciendo diferentes modificaciones sobre la estructura de las proteínas, afectando la actividad y función de las enzimas, hormonas y proteínas de transporte, haciéndolas sensibles a la proteólisis. La oxidación de los ácidos grasos, provoca la lipoperoxidación, que produce una pérdida de permeabilidad en la membrana celular además de que esta se vuelve frágil, lo que incrementa la lisis celular. Mientras que su acción sobre los ácidos nucleicos puede provocar la ruptura de una o ambas hebras del ácido desoxirribonucleico (ADN) incrementando la incidencia de las mutaciones además de producir cambios en los perfiles de metilación modificando la programación del epigenoma ${ }^{3,14,15}$, en ambos casos estas alteraciones pueden pasar a las siguientes generaciones. Así, el EOx afecta los mecanismos de reparación celular y el sistema de control inmunológico ${ }^{16}$, dañando a los tejidos y órganos, por lo que éste se encuentra involucrado en el desarrollo de diversas enfermedades, entre ellas la diabetes, obesidad, hipertensión, cáncer y otras, donde además por el mismo estado fisiológico se incrementa, la producción de las ERO intensificando el daño oxidativo ${ }^{3}$. 
Tabla 1. 20 principales países con mayor índice de contagios de COVID-19

Tomado y adaptado de WHO Coronavirus Disease (COVID-19) Dashboard (https://covid19.who.int/table), actualizado el 18/02/2021.

\begin{tabular}{|c|c|c|c|c|c|c|}
\hline \multirow[t]{2}{*}{ Posición } & País & \multicolumn{2}{|c|}{ Total de casos } & \multicolumn{2}{|c|}{ Total de muertes } & \multirow{2}{*}{$\begin{array}{c}\text { Probabilidad } \\
\text { de muertes } \\
\text { acumuladas } \\
\text { (\%) }\end{array}$} \\
\hline & & acumulados & en 24 horas & acumuladas & en 24 horas & \\
\hline 1. & Estados Unidos & $27,491,574.00$ & $57,856.00$ & $\begin{array}{r}484,379.0 \\
0\end{array}$ & 1390 & 1.76 \\
\hline 2. & India & $10,950,201.00$ & $12,881.00$ & $\begin{array}{r}156,014.0 \\
0\end{array}$ & 101 & 1.42 \\
\hline 3. & Brasil & $9,921,981.00$ & $55,271.00$ & $\begin{array}{r}240,940.0 \\
0\end{array}$ & 1167 & 2.43 \\
\hline 4. & Federación Rusa & $4,125,598.00$ & $13,447.00$ & $81,926.00$ & 480 & 1.99 \\
\hline 5. & Reino unido & $4,071,189.00$ & $12,717.00$ & $\begin{array}{r}118,933.0 \\
0\end{array}$ & 738 & 2.92 \\
\hline 6. & Francia & $3,453,645.00$ & $23,919.00$ & $82,692.00$ & 309 & 2.39 \\
\hline 7. & España & $3,107,172.00$ & $4,691.00$ & $66,316.00$ & 43 & 2.13 \\
\hline 8. & Italia & $2,751,657.00$ & $12,066.00$ & $94,540.00$ & 369 & 3.44 \\
\hline 9. & Turquía & $2,609,359.00$ & $7,325.00$ & $27,738.00$ & 86 & 1.06 \\
\hline 10. & Alemania & $2,360,606.00$ & $10,207.00$ & $66,698.00$ & 534 & 2.83 \\
\hline 11. & Colombia & $2,202,598.00$ & $4,049.00$ & $57,949.00$ & 163 & 2.63 \\
\hline 12. & Argentina & $2,033,060.00$ & $4,003.00$ & $50,432.00$ & 105 & 2.48 \\
\hline 13. & México & $2,004,575.00$ & $8,683.00$ & $\begin{array}{r}175,986.0 \\
0\end{array}$ & 1329 & 8.78 \\
\hline 14. & Polonia & $1,614,446.00$ & $9,074.00$ & $41,582.00$ & 274 & 2.58 \\
\hline 15. & Irán & $1,542,076.00$ & $8,042.00$ & $59,184.00$ & 67 & 3.84 \\
\hline 16. & África del sur & $1,496,439.00$ & $2,320.00$ & $48,478.00$ & 165 & 3.24 \\
\hline 17. & Ucrania & $1,287,141.00$ & $6,237.00$ & $24,852.00$ & 163 & 1.93 \\
\hline 18. & Perú & $1,244,729.00$ & $6,228.00$ & $44,056.00$ & 176 & 3.54 \\
\hline 19. & Indonesia & $1,243,646.00$ & $9,687.00$ & $33,788.00$ & 192 & 2.72 \\
\hline 20. & Republica Checa & $1,123,252.00$ & $10,930.00$ & $18,739.00$ & 143 & 1.67 \\
\hline
\end{tabular}

\section{EI SAR-coV-2}

EI SAR-coV-2, es un virus de ARN que en su genoma posee información para codificar proteínas estructurales de la envoltura $(E)$, la membrana $(M)$, la nucleoproteína (N) y la glicoproteína espiga (S) (Figura 1). Siendo ésta última (proteína S), la clave para la infección del virus, debido a que esta proteína se une a la proteína receptora ACE2 (enzima convertidora de angiotensina 2) de la célula huésped, la cual introduce al virus a la célula, donde inicia su replicación. Si bien la cepa original del virus inició en China, este ha adquirido diferentes mutaciones, principalmente en la región codificadora del gen $S{ }^{17}$, lo que complicó la obtención de una vacuna. Actualmente se han reportado diferentes tipos de SARcoV-2, donde cada uno produce diferente sintomatología. Uno de estos tipos de virus, representan el $1.5 \%$ de las personas infectadas los cuales presentan dolor de cabeza, pérdida de olfato, dolores musculares, tos, dolor de garganta, dolor de pecho y no presentan fiebre.
Mientras que otra variante del virus, se presenta en el $19.8 \%$ de las personas infectadas quienes presentan dolor de cabeza, pérdida de olfato, pérdida de apetito, tos, fiebre, ronquera, dolor de garganta, dolor de pecho, fatiga, confusión, dolor muscular, falta de aliento, diarrea y dolor abdominal, siendo estas últimas las personas que tienen mayor probabilidad de ser hospitalizadas. Por lo tanto, la sintomatología puede ser un factor para reconocer a las personas que necesitan mayor atención médica ${ }^{18}$.

\section{ACE2 regulador del estrés oxidativo}

La enzima ACE2, cataliza la conversión de angiotensina 2 , en angiotensina ${ }^{1,19}$, se expresa en 72 órganos distintos, incluyendo corazón, intestino, riñones y cerebro, donde se encuentra abundantemente en las células epiteliales del intestino delgado y del pulmón ${ }^{20}$. La angiotensina 2, tiene la función de vasoconstrictor y reabsorción de sodio, también participa en los procesos 
inflamatorios induciendo expresión de la proteína Nicotiamida Adenina Dinucleotido Fosfato oxidasa (NADPH oxidasa), la cual produce ERO, que al incrementar sus niveles produce un estado de EOx dando lugar a la desmetilación del gen ACE2, induciendo una sobreexpresión de esta proteína lo que lleva a un incremento de receptores del virus en membrana celular, aumentando la tasa de infección celular ${ }^{15}$. Cuando el virus se encuentra dentro de la célula, se amplifica la respuesta inflamatoria, mediante la liberación de citocinas ${ }^{16}$, produciendo un ambiente altamente oxidativo, dañando las estructuras y componentes celulares, promoviendo la replicación viral e induciendo apoptosis para la liberación de los nuevos virus ${ }^{21}$, lo que resulta, principalmente en un daño irreparable del pulmón.

Por otro lado, es importante notar que la desmetilación del gen ACE2 produce una modificación del epigenoma, la cual puede ser heredable, haciendo a las futuras generaciones probablemente más susceptibles a la infección de SAR-coV-2 o algún otro coronavirus.

\section{Importancia de la nutrición en el desarrollo de COVID-19}

En México, una gran parte de la población presenta síndrome metabólico (SM), en el 2018, la estimación de muertes por este

síndrome fue del $40 \%$ 22. El SM, se encuentra conformado por

tres patologías que son diabetes, hipertensión y obesidad ${ }^{23}$, siendo esta última la considerada como el factor principal en el desarrollo del SM. En México presenta una prevalencia mayor en mujeres que en hombres, ${ }^{24}$. Si bien, existe una predisposición genética, actualmente tienen su origen en la mala alimentación. Pese a que ya desde hace muchos años se viene tratando el tema del efecto dañino de los alimentos chatarra, bebidas azucaradas, y alimentos altos en grasa en la prevalencia

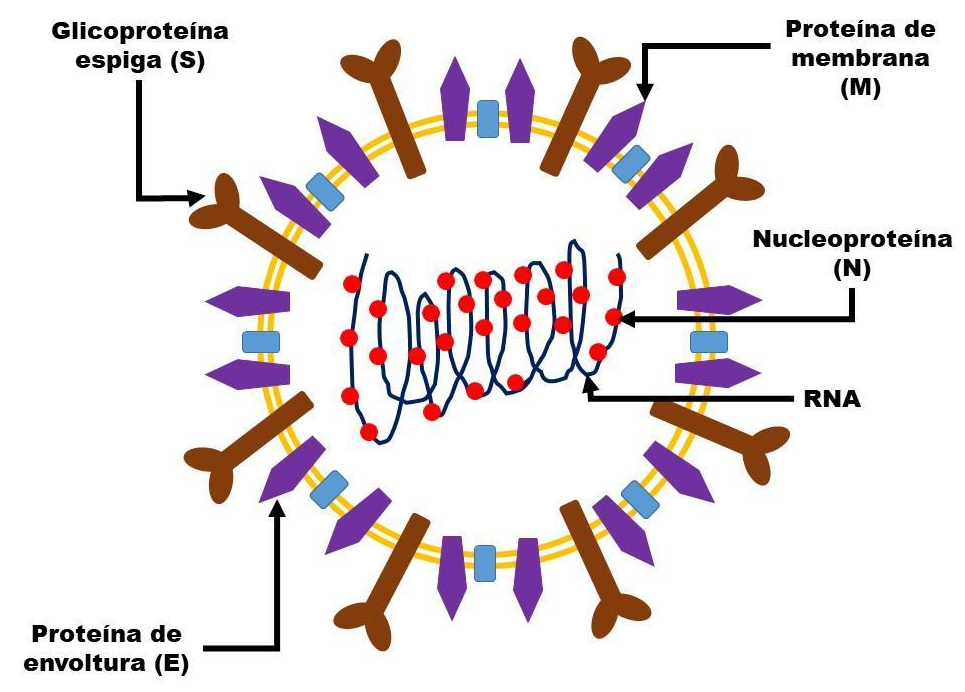

Figura 1. Estructura del SAR-coV-2. EL virus está formado por una membrana lipídica donde se encuentran incrustadas las proteínas de envoltura (E), de membrana (M) y la glicoproteína espiga (S), la cual se une a la proteína ACE2.

de la obesidad y otras enfermedades asociadas, éstos siguen predominando en la dieta mexicana, donde por otro lado, existe un bajo consumo de frutas y verduras ${ }^{25}$, las cuales son ricas en antioxidantes. Si a esto le sumamos la poca actividad física que se realiza, la cual ahora es casi nula debido a la actual pandemia, el índice de enfermedades asociadas al SM podría estar aumentando en la población, haciéndola más susceptible al desarrollo de diversas enfermedades, lo que aumenta la probabilidad de que esta población se infecte con SARcoV-2. Por lo que sería importante, ante la situación actual, difundir entre la población programas de buenos hábitos alimenticios y actividad física, que lleven a mejorar el estado de salud de la población, a fin de evitar nuevas infecciones.

Es muy importante considerar que la ingesta de una dieta balanceada aporta todos los nutrientes necesarios para el óptimo funcionamiento del organismo, como los elementos traza ( $\mathrm{Zn}, \mathrm{Cu}, \mathrm{Mg})$, los cuales incrementan la resistencia a las infecciones virales, fortaleciendo el sistema inmune, incrementando la síntesis de inmunoglobulinas y uniéndose a los antioxidantes para que éstos sean activos. Las vitaminas, al igual que los minerales pueden participar fortaleciendo el sistema inmune, además de que algunas actúan también como antioxidantes. Los ácidos grasos de cadena larga, sobre todo los omega-3 y 6 participan en la modulación del sistema inmune y control de los procesos inflamatorios ${ }^{26}$.

\section{Terapia antioxidante}

EI EOx juega un papel importante en la infección del SARcoV-2, por lo que resalta la importancia de analizar el posible uso de antioxidantes en el tratamiento y prevención del COVID-19. Si bien existen pocos estudios sobre este temad de frontera, se ha reportado que la 
administración vía intravenosa de 10-20 g diarios de la vitamina $\mathrm{C}$, divididos en periodos de 8 a $10 \mathrm{~h}$, en pacientes con COVID-19 conectados a respiración mecánica, produce una disminución de la lesión pulmonar inflamatoria aguda produciendo una mejoría en todos ellos. Lo cual se debe a que esta vitamina reduce a la NADPH oxidasa 2 (Nox2), disminuyendo la producción de las ERO 27, 28.

Así mismo, la vitamina $D$ inhibe la secreción de citocinas, con lo que se reduce la inflamación pulmonar, también estimula la síntesis de interleucina 10, aumenta la expresión de la Glucosa-6-fosfato deshidrogenasa (G6PD) e incrementa los niveles de glutatión oxidado, el cual interviene en la reducción de la NADPH evitando la producción del EOx, por lo que esta vitamina podría proteger el sistema respiratorio a través de sus efectos antioxidantes y antiinflamatorios ${ }^{29}$.

Por otro lado, se ha propuesto el uso de melatonina en el tratamiento de COVID-19. Esta es una hormona que se encuentra presente en plantas y animales, incluyendo a los humanos, donde participa en diversos procesos celulares. Es producida durante la noche, mientras que en el día sus niveles disminuyen, presenta efectos antioxidantes y además estimula el sistema antioxidante endógeno ${ }^{30}$. Las pruebas en animales muestran que esta hormona inhibe al inflamasoma NLRP3, el cual participa en la producción de neumonía, además de que puede prevenir la fibrosis, ambas patologías asociada a COVID19, lo que podría prolongar la vida del paciente. Además de que podría ser utilizada como coadyuvante para intensificar la acción de los fármacos actualmente utilizados ${ }^{29,31}$.

Otro antioxidante analizado es la catalasa, la cual es un antioxidante endógeno, pero que también se encuentra presente en tubérculos como el rábano. Pruebas en Rhesus macaques mostraron que su uso provoca una regulación en la producción de citocinas, además de prevenir la replicación del SAR-coV-2 ${ }^{21}$. Por otro lado, existen diversos estudios donde se emplean diversos antioxidantes para combatir a las ENT, los cuales podrían probarse en el tratamiento y prevención de COVID-19.

\section{Conclusiones y Recomendaciones}

La actual crisis mundial provocada por COVID-19, ha paralizado prácticamente a todo el mundo, afectando la economía, la vida social y la salud física y mental de las personas. Este virus ha cobrado miles de vidas, mientras que las infecciones continúan poniendo en riesgo nuestro futuro. Es importante seguir las recomendaciones de distanciamiento social y uso de cubrebocas, sin embargo, no toda la población lo hace, lo que ha contribuido a la propagación del virus. Otro factor que hay que tomar en cuenta, son las personas asintomáticas que son personas aparentemente sanas, pero con posibilidad de infectar a otras personas.

El estrés oxidativo está implicado en la infección de SARcoV-2, a través de la inducción de la expresión de la proteína ACE2, mediante un mecanismo epigenético, el cual puede tener implicaciones a largo plazo, por lo que sería importante evaluar los cambios en el epigenoma producidos en las personas que se han recuperado de
COVID-19, a fin de prevenir nuevas infecciones en un futuro.

En conclusión, debido a que el EOx está implicado en la infección de SAR-coV-2, por lo que una forma de prevenir el desarrollo de COVID-19, es llevar una alimentación balanceada. Disminuir la ingesta de comida chatarra, bebidas azucaradas y alimentos altos en grasa, incluyendo en la dieta frutas y verduras ricas en minerales y antioxidantes, e incluso tomar suplementos con estos compuestos. Además, incrementar el consumo de ácidos grasos omega 3 y omega 6 , así como realizar algún tipo de ejercicio, para ayuda a reducir el estrés oxidativo y fortalecer el sistema inmune.

Sin embargo, una vez que se presenta la infección, la alimentación no es suficiente para lograr revertir el daño. Sin embargo. la administración de compuestos antioxidantes ha mostrado tener algún efecto positivo. En esta perspectiva, aún es necesario realizar más investigaciones acerca del tema.

\section{Referencias}

[1] Ramírez-Ortiz J, Castro-Quintero D, Lerma-Córdoba C, Yela-Ceballos F, Escobar-Córdoba F. Consecuencias de la pandemia COVID 19 en la salud mental asociadas al aislamiento social. Colombian Journal of Anesthesiology. 2020; 48. e930.

[2] HHS. Centro para el control y prevención de enfermedades. (2020). Enfermedad del Coronavirus (COVID-19). USA. Recuperado el 20 /11/2020, de: https://espanol.cdc.gov/coronavirus/2019-ncov/needextra-precautions/people-with-medical-conditions.html

[3] Delgado Olivares L; Betanzos G; Sumaya-Martínez MT. Importancia de los antioxidantes dietarios en la disminución del estrés oxidativo. Investigación y Ciencia de la Universidad de Aguascalientes. 2010; 50: $10-15$.

[4] Peña-Oyarzun D, Bravo-Sagua R, Diaz-Vega A, Aleman L, Chiong M, Garcia L, Bambs C, Troncoso R, Cifuentes M, Morselli E, Ferreccio C, Quest AFG, Criollo A, Lavandero S. Autophagy and oxidative stress in non-communicable diseases: A matter of the inflammatory state? Free Radic Biol Med. 2018; 20(124):61-78.

[5] de Las Heras N, Martín Giménez VM, Ferder L, Manucha W, Lahera V. Implications of Oxidative Stress and Potential Role of Mitochondrial Dysfunction in COVID-19: Therapeutic Effects of $\begin{array}{llll}\text { Vitamin D. } & \text { Antioxidants. }\end{array}$ https://doi.org/10.3390/antiox9090897

[6] Ioannidis J, Axfors C, Contopoulos-Ioannidis D. Population-level COVID-19 mortality risk for non-elderly individuals overall and for non-elderly individuals without underlying diseases in pandemic epicentres. Environmental Research. 2020; 188:109890. https://doi: 10.1016/j.envres.2020.109890.

[7] World Health Organization (OMS). Coronavirus Disease (COVID-19) Dashboard. Recuperado el 18/02/2021, de: https://covid19.who.int/table

[8] Observatorio Mexicano de Enfermedades No Transmisibles (OMENT). Mortalidad por enfermedades no transmisibles en México. Recuperado el 18/02/2021, de: http://oment.salud.gob.mx/aumentan-en-mexicomuertes-relacionadas-con-enfermedades-no-transmisibles/

[9] World Health Organization (OMS), Noncommunicable diseases Recuperado el 18/02/2021, de: https://www.who.int/news-room/factsheets/detail/noncommunicable-diseases

[10] Laurencin C T, McClinton A. (2020). The COVID-19 Pandemic: a Call to Action to Identify and Address Racial and Ethnic Disparities. Journal of racial and ethnic health disparities. 2020; 7(3), 398-402. 
[11] Fouad M N, Ruffin J, Vickers S M. COVID-19 Is Disproportionately High in African Americans. This Will Come as No Surprise...The American Journal of Medicine. 2020; 133(10): E544-E545.

[12] Instituto Nacional de Salud Pública. Encuesta Nacional de Salud y Nutrición 2018 (ESANUT 2018). Recuperado de: el 15/02/2021, de: https://ensanut.insp.mx/encuestas/ensanut2018/doctos/informes/ ensanut_2018_presentacion_resultados.pdf

[13] Shampa Chatterjee, Chapter Two - Oxidative Stress, Inflammation, and Disease. In Thomas Dziubla, D. Allan Butterfield, Oxidative Stress and Biomaterials. Academic Press, Massachusetts. 2016. Pages $35-58$,

[14] de Araújo R, Bruneska D, Borba MA. Oxidative Stress and disease. Chapter 10. In: Morales-Gonzalez, J.A., Morales-Gonzalez, A., Madrigal-Santillan, E.O. A Master Regulator of Oxidative stress - the Transcription Factor Nrf2. InTech. London. 2016. Pages. 185-199.

[15] Shawalha AH, Zhao M, Coit P, Lu Q. Epigenetic dysregulation of ACE2 and interferon-regulated genes might suggest increased COVID19 susceptibility and severity in lupus patients. Clinical Immunology. 2020; 215: 1-4.

[16] Derouiche S. Oxidative Stress Associated with SARS-Cov-2 (COVID19) Increases the Severity of the Lung Disease - A Systematic Review. Journal of Infectious Diseases and Epidemioly. 2020; 6:121. https://doi: 10.23937/2474-3658/1510121

[17] Yin C. Genotyping coronavirus SARS-CoV-2: methods and implications. Genomics. 2020; 112(5): 3588-3596.

[18] Sudre C, Lee K, Lochlainn M, Varsavsky T, Murray B, Graham M, et al. Symptom clusters in Covid19: A potential clinical prediction tool from the COVID Symptom study app. Med Rxiv. 2020; 06.12.20129056. https://doi.org/10.1101/2020.06.12.20129056

[19] Haleem A, Javaid M, Vaishya R. Effects of COVID 19 pandemic in daily life. Current medicine research and practice. 2020; 10(2): 78-79.

[20] Hamming I, Timens W, Bulthuis M L, Lely, A T, Navis G, van Goor $\mathrm{H}$. Tissue distribution of ACE2 protein, the functional receptor for SARS coronavirus. A first step in understanding SARS pathogenesis. The Journal of pathology. 2004; 203(2): 631-637.

[21] Qin M, Cao Z, Wen J, Yu Q, Liu C, Wang F, et al. An Antioxidant Enzyme Therapeutic for COVID-19. Advances Materials. 2020; 32(43) 2004901. https://doi.org/10.1002/adma.202004901

[22] Aceves E, Ángeles A. Jiménez F. Desentrañando el síndrome metabólico. (2019). Recuperado el 13/02/2020, de: https://www.c3.unam.mx/boletines/boletin35.html

[23] Baez B.G, Pérez R, Zamora I, Torres E, Ruiz G, Nieva A. Síndrome metabólico y su relación con estrés oxidativo. Diabetes Hoy para el Médico y el profesional de la Salud. 2011; 12(5): 2814-2822.

[24] Campos I, Cuevas L, González L, Hernández L, Shamah T, González T, Rivera J. Epidemiología de la obesidad y sus principales comorbilidades en México. En Rivera J, Colchero M, Fuentes M, González T, Aguilar A, Hernández G, Barquera S. La obesidad en México. Instituto Nacional de Salud Pública. México. 2018.Páginas 3140.

[25] Batis C, Sánchez T, García C, Rodríguez S, Ramírez I. Dieta en México y efectos salud. En Rivera J, Colchero M, Fuentes M, González T, Aguilar A, Hernández G, Barquera S. La obesidad en México. Instituto Nacional de Salud Pública. México. 2018. Páginas 41-52

[26] Jaggers G K, Watkins B A, Rodríguez R L. (2020). COVID-19: Repositioning nutrition research for the next pandemic. Nutrition Research. 2020; 81:1-6.

[27] Loffredo L, Violi F. (2020. COVID-19 and cardiovascular injury: A role for oxidative stress and antioxidant treatment? International Journal of Cardiology. 2020; 1(312):136. https://doi: 10.1016/j.ijcard.2020.04.066.
[28] Cheng R. Z. Can early and high intravenous dose of vitamin C prevent and treat coronavirus disease 2019 (COVID-19)? Medicine in Drug Discovery. 2020; 5: 100028 https:/doi: 10.1016/j.medidd.2020.100028

[29] Giménez V M, Inserra F, Tajer C D, Mariani J, Ferder L, Reiter R J, et al. Lungs as target of COVID-19 infection: Protective common molecular mechanisms of vitamin D and melatonin as a new potential synergistic treatment. Life sciences. 2020; 254, 117808. https://doi: 10.1016/j.lfs.2020.117808

[30] Reyes-Prieto B M, Velázquez-Paniagua M, Prieto-Gómez B. Melatonina y Neuropatologías. Revista de la Facultad de Medicina. 2009; 52: 105-109.

[31] Zhang R, Wang X, Ni L, et al. COVID-19: Melatonin as a potential adjuvant treatment. Life Sci. 2020; 250,117583. https://doi.org/10.1016/j.lfs.2020.117583 